\title{
El proceso de la familia en torno a la menopausia de la mujer
}

\author{
Rosa Ofelia Alvarez de S.*
}

RESUMEN: Durante la época de la menopausia de la madre, ocurre en la familia una serie de cambios en sus diferentes miembros, los cuales se analizan en este artículo, con el fin de dar una visión integral de estos procesos, ampliar el marco de referencia y posibilitar un manejo adecuado de las dificultades. Los cambios que se presentan en la menopausia están influenciados por varios factores tales como las concepciones culturales, que cambian continuamente por las transformaciones que ha presentado la familia durante este siglo, en relación con las etapas prefilial, filial y postfilial y donde el papel que adquiere la pareja ha inducido a considerarla como un sistema independiente. En esta época coexisten con la menopausia en la mujer, la andropausia en el hombre, la adolescencia y/o salida de los hijos, la vejez y muerte de los abuelos, la jubilación laboral de cada uno de los miembros de la pareja y además las enfermedades cuyos riesgos son más frecuentes en este período.

La familia debe conocer los procesos y efectuar una serie de ajustes y concertaciones con el fin de manejar adecuadamente los problemas, mediante una comunicación clara, abierta y directa, además con tolerancia y aceptación entre sus miembros, que permitan un desarrollo funcional y armónico de ella y de cada uno.

Se analizaron los subsistemas parentofilial, fraterno e igualmente algunos de los suprasistemas.

PALABRAS ClAVES: Familia, Menopausia, Andropausia, Teoría de Sistemas.

SUMMARY: During the mother's menopause several changes in different members of the family are developed. These changes are analyzed in order to give a whole view of them, to wide the reference frame and to make possible an accurate management of the difficulties.

Women's menopausal changes are influenced by several factors such as the cultural conceptions which are changing continuously because of the family transformation during the present century related with the prefilial, filial and postfilial stage and where the role of de couple has induced to consider it as an independent system.

At this time with mother's menopause, coexist mate's andropause, children's adolescence and homeleaving, grand parents oldness and death and additionally, the job retirement of each member of the couple, similarly the risk of certain deseases is increased.

Family most know such processes and make several agreements and adjustments in order to accurately the problems by means of a clear, open and direct communication besides the tolerance and acceptation between family members which will permit a functional and harmonic development of it and each one.

The parent filial and fraternal subsystems and also some suprasystems are analysed.

KEY WORDS: Family, menopause, andropause, systems theory.

Los avances científicos y el progreso alcanzado a nivel mundial, han permitido que se disminuya la mortalidad infantil y se aumente la expectativa de vida, fenómeno que también ocurre en nuestro país, según el Dane, en el Boletín del Instituto Nacional de Salud de 1993, se aprecia como la expectativa de vida al nacer viene en aumento:

\begin{tabular}{|c|c|c|}
\hline Año & Población ajustable & Esperanza al nacer \\
\hline \hline 1985 & $29^{\prime} 879.330$ & 67.16 \\
\hline 1990 & $32^{\prime} 978.170$ & 69.26 \\
\hline 1995 & $36^{\prime} 181.860$ & 70.23 \\
\hline 2000 & $39^{\prime} 397.190$ & 72.05 \\
\hline 2015 & $48^{\prime} 647.060$ & 74.29 \\
\hline
\end{tabular}

* $\quad$ Médica. Especialista en Familia - Medellín.
Situación que trae como resultado el aumento de la expectativa de vida también en la mujer y por lo tanto una gran mayoría de ellas vivirán cerca de una tercera parte de su vida en menopausia, hecho que hace necesario que se emprendan investigaciones y estudios acerca de los fenómenos que se presentan durante este período de la vida de la mujer, con miras a lograr no sólo mayor expectativa, sino que ella sea de buena calidad y así lograr vivir más activas y productivas por mayor tiempo, para beneficio no sólo de la mujer sino también de su familia, sus amigos y de la sociedad en general.

\section{La influencia de la cultura en la menopausia}

El impacto que produce la menopausia en la familia está determinado por varios factores, uno muy importante se deriva de los conceptos, creencias y mitos que se han adquirido a través de la cultura por medio de los 
procesos de socialización, hecho que crea expectativas y temores en todos sus miembros, y que es necesario hacerlos explícitos con el fin de poder analizarlos, discutirlos, y aceptarlos o rechazarlos según sea el caso, ya que las realidades que se han aprendido e introyectado como absolutas e indiscutibles, son sólo explicaciones aprendidas en ese proceso socializador, las cuales podemos modificar al encontrar nuevas explicaciones coherentes con nuestras experiencias vivenciales. Por ejemplo, al saber que una persona está en menopausia, ¿cuáles son los pensamientos que se tienen acerca de lo que ella está viviendo? Esa respuesta que brota casi automáticamente es el producto de los conceptos introyectados en el individuo a través de la cultura, en donde predominan las connotaciones negativas en nuestro contenido ideológico tal como: inutilidad, mal genio, irritabilidad, pérdida de la sexualidad, masculinización, vejez y muerte; pero cuantas veces en nuestra cotidianidad se encuentran mujeres en este período que son seguras, dueñas de sí, autónomas y productivas, satisfechas, femeninas, bellas física, emocional y espiritualmente, con capacidad de compartir y de proyectarse a la comunidad.

Por lo anteriormente expuesto, se hace necesario un replanteamiento de los conceptos adquiridos a través de la cultura, con el fin de modificar los conceptos negativos mediante los conocimientos científicos, recursos y nuevos modelos y capitalizar los hechos positivos que genera este momento.

\section{Concepto de familia}

Es necesario modificar el concepto estático y tradicional de familia, cual es la conformada por papá, mamá e hijos, pues ya no es suficiente para englobar los cambios que se presentan en la sociedad actual y por lo tanto es necesario tener un concepto más amplio donde puedan tener cabida todas las tipologías de familia que a diario se están observando.

Entonces qué es una FAMILIA? Según Gabriel Smilkstein ${ }^{1}$ :

"La familia es una unidad básica de la sociedad en la cual dos o más adultos, con o sin niños o también un adulto con niños tienen el compromiso de nutrirse o apoyarse emocional y físicamente. Deciden compartir recursos tales como el tiempo, espacio y dinero. Los miembros de la familia frecuentemente funcionan en un lugar donde hay una sensación de hogar".

Nos situaremos en el marco epistemológico que permite ver a la familia como un sistema ${ }^{2}$, la cual está compuesta por varios subsistemas tales como el conyugal, o sea el conformado por la pareja; el parentofilial y se refiere al que se forma en la relación padres e hijos; el fraterno entre hermanos; también el subsistema compuesto por los hombres y el compuesto por las mujeres,

MEJIA, D. y Cols. Salud Familiar. Sistema Educación Continua. Instituto de Seguros Sociales y Asociación Colombiana de Facultades de Medicina. Primera Edición. Bogotá. Octubre de 1990. pág. 30.

2 ANDOLFI, Maurizio. Terapia Familiar. 1 a edición. Buenos Aires. 1984. p. 17. el de los hijos mayores y el de los menores, etc.; subsistemas que interactúan entre sí y se afectan recíprocamente, bien sea positiva o negativamente en el quehacer cotidiano. A la vez, estos subsistemas están inmersos en otros suprasistemas y que corresponden a las diferentes redes sociales, tales como los conformados en el trabajo, los amigos, los deportes, la iglesia, los sitios donde se administra justicia, etc. y que además se vuelven fuente de recursos cuando hay problemas o dificultades.

Recientemente, se concibe a la pareja ${ }^{3}$ como sistema independiente de los subsistemas de la familia, concepto nuevo que le da vida autónoma a la pareja, este sistema es necesario y fundamental en el funcionamiento familiar, es muy importante que sea fuerte para que puedan desempeñar el papel de padres en forma adecuada, fácil y armónicamente. Este concepto del sistema conyugal independiente de la familia permite comprender y manejar en forma clara las situaciones de ruptura del sistema de la pareja, por separación o divorcio, para que no conlleven a la concepción de desintegración familiar que existe en nuestra cultura, pues los otros subsistemas (parento-filial, fraterno, etc.) pueden y deben permanecer interactuando, ya que el vínculo adquirido con la paternidad no desaparece al disolverse la relación conyugal.

\section{Evolución de la familia a través del tiempo}

La familia durante su evolución generalmente pasa por tres épocas: a) Prefilial o período anterior a la llegada de los hijos. b) Filial que comprende desde la llegada de los hijos hasta que ellos abandonan el hogar. y c)Postfilial desde el momento en que la pareja nuevamente sola, se reencuentra consigo misma al ausentarse los hijos ${ }^{4}$.

Hasta hace poco el período filial comprendía el mayor tiempo de la familia como consecuencia del gran número de hijos que alcanzaban a tener: 10, 15 y hasta 20, por lo cual todo el tiempo lo pasaba la pareja nutriendo y educando a los hijos y cuando los últimos abandonaban el hogar, ya uno de los padres o los dos habían muerto, debido a que las expectativas de vida era menor de 50 años, por lo anterior era muy corta o no existía la etapa postfilial; la etapa prefilial también era corta debido a que las parejas iban con el deseo de tener rápidamente los hijos, máxime que la mujer permanecía mucho tiempo de su vida en el hogar, no utilizaban métodos anticonceptivos y además sus creencias religiosas así se lo exigían.

En el momento del ciclo vital de la familia, cuando uno de los miembros está en menopausia, coexiste en la dinámica familiar una serie de eventos muy importantes de considerar con relación a cada uno de los miembros que la conforman, tanto para la pareja como en cada uno de los hijos. Concepto emitido por Fernando Coddou y Carmen Luz Méndez
en el Seminario Taller Terapia de Pareja organizado por la Facul-
tad de Medicina de la Universidad de Antioquia, Educación
Médica, en Medellín, Septiembre 15 a 17 de 1994 .
COBOS, Francisco. Familia: Introducción a la Psicoterapia Fami-
liar. Ed. Universidad Nacional de Colombia. Bogotá(1986). p. 46. 


\section{La familia en la época actual}

La conformación de la familia en la época moderna está centrada en la pareja, pues el Periodo prefilial y postfilial es mucho más prolongado, y por lo tanto el tiempo disponible para la convivencia en pareja se amplía y aquél que ocupan los hijos: etapa filial, es cada vez. más corto, o a veces no existe, como consecuencia de que las parejas tienen tendencia a contraer matrimonio más tardíamente, pues gastan parte de su tiempo en su educación y preparación, se hace necesario que ambos cónyuges trabajen para el sostenimiento del hogar; se demoran un tiempo prudencial para tener los hijos mientras se estabilizan económicamente y cuando deciden tenerlos, sólo conciben de uno a tres, los cuales crecen, se educan y salen del hogar más rápidamente. La etapapostfilial se prolonga no sólo por la salida rápida de los hijos sino también por la prolongación de la expectativa de vida, tiempo que la pareja debe utilizar para disfrutar los últimos años de su vida.

Durante la prolongación de la etapa postfilial coexisten algunos fenómenos, es así como cuando la mujer entra en la menopausia, o período de cese de sus menstruaciones como resultado de la refractariedad del ovario a las gonadotrofinas, también el hombre está presentando un sinnúmero de cambios, generalmente está en el período de la jubilación y las oportunidades de trabajo y remuneración son cada vez más escasas, como también disminuye su rendimiento laboral. Su estado de salud empieza a deteriorarse, máxime si no ha tenido interés en cuidarse en épocas anteriores, y como consecuencia se aumentan sus factores de riesgo (cigarrillo, alcohol, obesidad), además ocurren cambios endocrinos tal como el déficit de esteroides sexuales que se conoce como andropausia; todo esto hace que se altere su autoestima.

Al mismo tiempo que se suceden estos fenómenos en el hombre, la mujer también está pasando por etapas difíciles tales como la jubilación en las que laboran fuera de la casa y cambios secundarios a las alteraciones hormonales, que pueden llevar a crisis y producir inestabilidad en el sistema de la pareja, generar una serie de cambios lo que hace necesario que se concerten dificultades con su compañero, con el fin de poder vivir felices los últimos años de sus vidas; cuando esto no sucede se producen conflictos que llevan a peleas y discusiones frecuentes y a veces los conducen hasta la separación.

Durante esta época, la comunicación en la pareja debe ser más clara, directa y adecuada, como producto de las habilidades adquiridas y del manejo de las dificultades servidas en el diario vivir y generadas por el crecimiento individual y de la familia durante su ciclo vital.

Cuando hay transtornos en la comunicación tales como: a) Incongruencia en el lenguaje verbal y no verbal, b) Comunicación confusa, c) Cuando la comunicación es indirecta, como la que se hace a través de terceros, por ejemplo: "dile a tu papá que no voy a salir con él”, y el papá responde con otro mensaje, d) Comunicación agresiva, y, e) Cuando la comunicación está bloqueada, en donde la comunicación verbal se suspende; todos estos transtornos generan dificultades en las interrelaciones del sistema de pareja, tanto en su funcionamiento interno, como también para desempeñar su rol de padres.
La forma de comunicarse se aprende en la familia y por lo tanto, los hijos podrán tener iguales aciertos o dificultades al comunicarse entre sí, con sus padres y con la sociedad misma, como lo aprendió en su familia.

En la etapa de la menopausia la sexualidad de la pareja está determinada principalmente por la satisfacción que cada uno siente de sí mismo y con el otro; pero también influyen factores biológicos que se presentan tanto en el hombre como en la mujer; es así como en la mujer los cambios secundarios a la insuficiencia ovárica y por lo tanto a la ausencia de estrógenos, causa irritabilidad, depresión, insomnio, lo que disminuye su autoestima y la disponibilidad para la relación sexual; a nivel genital produce atrofia de los epitelios y disminución de la lubricación vaginal durante la relación sexual, esto provoca dolor y ardor durante el coito; en el hombre se encuentra pérdida de la erección y dificultades en la eyaculación como también disminución o ausencia de espermatozoides, con la consecuente infertilidad secundaria, fenómeno que es el resultado de la disminución de la función testicular y de testosterona: andropausia. Hechos que traen como consecuencia la disminución de las relaciones sexuales y pérdida del orgasmo, estas situaciones pueden superarse con un adecuado manejo y orientación médica tal como el reemplazo hormonal, como también orientar a la pareja sobre el proceso que están viviendo.

La disfunción sexual de la pareja se agravará como resultado de los tratamientos médicos que reciben debido a las enfermedades que se presentan durante esta edad, tal como enfermedades cardiovasculares como la hipertensión, metabólicas como la diabetes y la obesidad, la osteoporosis y los estados depresivos, situación que además de los medicamentos afectan el ejercicio de la sexualidad tanto en el hombre como en la mujer. Hay que recalcar que estudios muestran la sexualidad en esta época como muy placentera y satisfactoria para muchas parejas que han logrado crecer y fortalecerse a lo largo de su vida y que los problemas físicos que se presentan se puedan manejar con una adecuada asesoría médica.

La alta frecuencia de separaciones en la relación de pareja durante este período, junto con el aumento en la mortalidad masculina por la menor expectativa de vida que él tiene, y agravado por el alto índice de violencia en nuestro medio, hace que la mujer tenga que asumir la jefatura y dirección del hogar, lo que aumenta la tensión en el sistema familiar y obliga a la redistribución de roles $y$ funciones en la familia.

\section{El subsistema parento-filial}

Otro reto interesante que enfrenta la familia durante la época de la menopausia de la mujer, es la presencia de hijos adolescentes y/o la salida de los hijos del hogar, llamada: "etapa del nido vacío" $5-6$.

\footnotetext{
5 CADAVID de G, Irene y OQUENDO M., Asceneth. La familia Humana. Un marco conceptual para el trabajo educativo preventivo con familias desde el enfoque sistémico. Tesis de grado. Facultad de Trabajo Social. Universidad Pontificia Bolivariana. Medellín. 1987. p. 90-99.

6 MINUCHIN, Salvador y FISHMAN, H. Ch. Técnicas de Terapia Familiar. Barcelona. Paidós. 1985. p. 39-40.
} 
La adolescencia, considerada como un período de grandes confrontaciones entre padres e hijos, dolorosa para ambos, por la dificultad de los primeros de entender y aceptar que los niños ya han crecido y se van volviendo adultos, autónomos y por lo tanto necesitan menos la presencia de los padres como en otros tiempos; y para los hijos aceptar el reto de volverse adultos y enfrentar todos los cambios físicos, sicológicos y sociales que el período de la adolescencia les exige. Es un momento donde se producen situaciones tirantes entre los miembros de estos subsistemas y aún en el sistema mismo de la pareja, lo que hace que en la familia se generen frecuentes conflictos y sus miembros se vuelvan vulnerables, pueden aparecer síntomas y la situación de tensión si no es manejada adecuadamente, puede llevar a la ruptura de la relación de pareja o acomodarse en una relación disfuncional en la familia.

Conocer los procesos que se están dando en la familia, tanto en unos como en los otros miembros, ayuda a disminuir la tensión y entender lo que ocurre; cuando la situación se torna difícil obligarían a la pareja o a la familia a buscar ayuda profesional, ayuda que tiene como fin evitar que se agraven los problemas o que queden heridas difíciles de borrar, además es el momento de capitalizar las soluciones a las dificultades en beneficio del crecimiento y enriquecimiento de la familia y sus miembros, situaciones que sirven de experiencia para manejar futuros problemas o dificultades en nuevas etapas de la vida.

La salida de los hijos es otra situación que perturba a la familia, ocurre en nuestro medio cuando los hijos se van a prestar el servicio militar, o salen a estudiar a otra ciudad, y en otros casos cuando contraen matrimonio o unión libre. Puede ser un momento gratificante para los padres cuando se prepararon y esperan ver sus hijos fuertes y capaces de enfrentar al mundo; en tanto para los que no han creado espacios propios que les permitan fortalecerse como pareja y que sólo piensan y viven en función de los hijos, se encontrarán abandonados y frustrados por la situación que les toca afrontar, en muchas oportunidades sin la solidaridad y apoyo del otro miembro de la pareja; en ocasiones los miembros de la pareja se comportan como dos extraños frente a frente, y por lo tanto será difícil reconstruir su relación.

El tiempo que disponía la pareja para sus hijos queda libre, tiempo que debe ser enriquecido en su convivencia y fortalecido, desarrollar actividades nuevas y recreación, lo que les permitirá aceptar con mayor facilidad la salida de los hijos y que ésta sea menos traumática.

\section{El subsistema fraterno}

Este subsistema, constituido por los hermanos de una familia los cuales interactúan entre sí, pero que al momento de la adolescencia, se desencadenan fuertes tensiones y controversias; es así como los mayores quieren conservar sus espacios y derechos adquiridos, a veces no comparten con los menores, estas situaciones de tensión alternan con momentos de amistad y armonía. Una de las principales dificultades que tiene el adolescente es el rechazo a los menores para evitar ser comparado o parecerse con un niño, pero en ocasiones temen asumir el papel de adultos. En esta época los adolescentes pasan el mayor tiempo con sus pares o iguales, en donde no sólo intercambian experiencias, lo que les permite afianzar su identidad, apoyarse y fortalecerse, y a la vez conocer la sociedad más a fondo, donde posteriormente se desenvolverán, en tanto los padres pierden el papel preponderante que tenían.

\section{La mujer en menopausia y los suprasistemas}

Al analizar lo que está sucediendo en la familia extensa cuando la mujer está en la menopausia, es importante tener en cuenta a los padres, tanto de ella como de su compañero, los abuelos desempeñan un papel muy importante en la familia, son fuente de la transmisión oral de la cultura, ricos en experiencias e historia y si los aprovechamos adecuadamente serán fuente de satisfacción para ella, sinembargo los abuelos tienen múltiples limitaciones físicas, sicológicas, económicas y espaciales lo que obligan a la hija, ya en menopausia, y a su compañero a tener que responder no sólo por los hijos sino también por los padres, "generación sánduche", por lo tanto a veces necesitan buscar recursos intra y extrafamiliares para dar un manejo adecuado a las situaciones económicas y afectivas que generan conflicto. La muerte de los abuelos también conmociona la familia y crea estrés.

Durante esta época, la familia sufre continuos cambios como consecuencia de la salida de miembros de la familia tal como los hijos o el padre o la madre en caso de divorcio de muerte; también la llegada de nuevos miembros tal como yernos, nueras y nietos, que obligan a unos y a otros a aceptar nuevos roles, redefinir funciones y aclarar límites para hacer de este hecho una situación satisfactoria para todos.

Los amigos de la pareja que generalmente están en este período, empiezan también a enfermarse o mueren, lo que plantea la necesidad de búsqueda de nuevas amistades con el fin de evitar la soledad y sus consecuencias.

\section{La incidencia de la menopausia en la mujer}

Todo este proceso que vive la familia durante esta época, se asocia a los cambios físicos y síquicos que presenta la mujer en la menopausia, la cual puede comprometer su salud de tal manera que no sólo estaría ella afectada sino también su familia.

La menopausia se produce como consecuencia de la pérdida de la función ovárica ${ }^{7}$, esto produce en ella una serie de signos y síntomas que se presentan a corto, mediano y largo plazo. Esta clasificación se hace más con un fin didáctico, pero en no raras ocasiones los síntomas y signos se superponen o varían en orden de aparición. estado fisiológico complejo. En: Acta Med. Colomb. Vol. 18 No. 5. Sep.-Oct 1993. p. 257-266. 
En estudios en población general, en una muestra no clínica y por lo tanto no seleccionada en consultorios médicos, se ha visto que el $85 \%$ de las mujeres son sintomáticas y de éstas sólo un $15 \%$ consultan al médico. Los síntomas más frecuentes que aparecen a corto plazo son los transtornos del ciclo previos al cese definitivo de la menstruación, éste último, es el primer síntoma que aparece y el que marca el inicio de la menopausia, está acompañado de la pérdida de la fertilidad; le siguen o anteceden las oleadas de calor y sudoración, cambios en los estados de ánimo como irritabilidad, depresión, nerviosismo, e insomnio.

A mediano plazo aparecen las atrofias como consecuencia de la pérdida paulatina del tejido colágeno y atrofia de los epitelios con la consecuente sequedad de piel y mucosas, lo que da origen a prurito generalizado, ardor ocular, escasa saliva, atrofia y caída de las mamas; atrofia en los genitales, ovarios, útero y vagina, pérdida de la lubricación, ardor y dolor postcoito; atrofia vulvar que desencadena ardor y prurito; el aparato urinario está comprometido con atrofia de la uretra y trígono, lo que produce síntomas tales como ardor al orinar (disuria), aumento en la frecuencia (polaquiuria y nicturia) en ocasiones facilita la invasión bacteriana con aumento de las infecciones urinarias.

El mayor problema se presenta con los síntomas a largo plazo tal como la osteoporosis y la arterioesclerosis, los cuales aparecen después de 10 ó 15 años de iniciada la menopausia.

La enfermedad cardiovascular aumenta en esta época hasta nivelarse en frecuencia con el hombre, y lo sobrepasa posteriormente; es causa importante en la mortalidad en la mujer, secundaria a hipertensión arterial, angina de pecho, infarto cardíaco y enfermedad cerebro vascular.
La osteoporosis, al igual que la enfermedad cardiovascular es multifactorial; las investigaciones han demostrado que la pérdida de los estrógenos precipitan la pérdida del tejido óseo, soporte del organismo y por lo tanto se aumenta su fragilidad, en donde pequeños traumas a veces desapercibidos, producen fracturas principalmente a nivel de muñeca, columna vertebral, con aumento de la cifosis normal, y la cadera, principalmente a nivel del cuello del fémur, lo que produce dolor, invalidez y en algunos casos hasta la muerte por complicaciones de estas fracturas o las cirugías necesarias.

En conclusión, es importante cuando se analiza a la mujer en la menopausia, evaluar no sólo los fenómenos que se suceden en ella en su condición física y síquica, sino también los fenómenos que se suceden a nivel de la familia nuclear en cada uno de los elementos que la constituyen, padres e hijos, como también en la familia extensa donde los abuelos, nueras, yernos y nietos, generan múltiples cambios, al entrar a ser parte de la familia, a compartir y a participar en la dinámica familiar.

Es necesario estimular a cada miembro de la familia para que logre un desarrollo armónico y satisfactorio, que le permita en cada etapa de la vida obtener las ventajas que se producen y manejar adecuadamente las dificultades que se presentan para continuar fortalecidos en la siguiente etapa del ciclo vital.

El conocimiento de esta dinámica familiar nos permite un manejo adecuado de las situaciones que se generan y evita sesgarnos al buscar sólo en la menopausia la única fuente de todos los problemas.

\section{BIBLIOGRAFIA}

1. Andolfi, Maurizio. Terapia Familiar. la edición. Buenos Aires. 1984. $174 \mathrm{p}$.

2. Cadavid de G, Irene y Oquendo M., Asceneth. La Familia Humana. Un marco conceptual para el trabajo educativo preventivo con familias desde el enfoque sistémico. Tesis de grado. Facultad de Trabajo Social. Universidad Pontificia Bolivariana. Medellín. 1987. 103 p.

3. Cobos, Francisco. Familia: Introducción a la Psicoterapia Familiar. Ed. Universidad Nacional de Colombia. Bogotá (1986); 115 p.
4. Mejía, Diego y Cols. Salud Familiar. Sistema Educación Continua. Instituto de Seguros Sociales y Asociación Colombiana de Facultades de Medicina. Primera Edición. Bogotá. Octubre de 1990. pág. 30.

5. Minuchin, Salvador y FISHMAN, H. Ch. Técnicas de Terapia Familiar. Barcelona. Paidós. 1985. 286 p.

6. Rodríguez, Kenny A. Onatra, William. La Menopausia: un estado fisiológico complejo. En: Acta. Med. Colomb. Vol. 18 No. 5. Sep.Oct. 1993. p. 257-266. 